\title{
THE NLC L-BAND BUNCH COMPRESSOR *
}

\author{
Zenghai Li, Paul Emma, Tor Raubenheimer, SLAC, USA
}

\section{Abstract}

The first stage bunch compressor in the NLC injector complex compresses the e+/e- beams from a bunch length of $5 \mathrm{~mm} \mathrm{rms}$ to $0.5 \mathrm{~mm} \mathrm{rms}$ at the beam energy of $2 \mathrm{GeV}$. To obtain this compression ratio, the compressor rf section operates with an rf frequency of $1.4 \mathrm{GHz}$ and a voltage of about $140 \mathrm{MV}$ while a magnetic wiggler is used to generate an $R_{56}=0.5 \mathrm{~m}$. The bunch compressor is designed to operate with a beam from the damping ring that has a bunch spacing slew of 20ps across the bunch train due to the transient loading in the damping rings. The compressor RF section is required to produce a specific energy profile along the bunch train so that the bunch spacing can be corrected in the compressor bending section. Further, the 1-amp beam heavily loads the compressor linac and beam loading compensation is essential to prevent a phase variation along the bunch train in the downstream linacs. In this paper, we will present simulation results of the beam loading compensation using a $\Delta \mathrm{T}$ scheme assuming various initial bunch spacing arrangements. We will study the impact of the different compressor energy profiles on the beam energy, energy spread, and bunch length at the IP.

\section{INTRODUCTION}

The NLC bunch compressor is a two-stage system [1] designed to compress each bunch of a 95-bunch train from a length of $5 \mathrm{~mm}$ at $2-\mathrm{GeV}$ damping ring (DR) extraction energy, to a $90-\mu \mathrm{m}$ rms bunch length at the beam interaction point (IP). The two-stage system includes L-band, S-band, and X-band RF components and has been designed to be relatively insensitive to extraction phase errors initiated in the damping rings [2]. The system is shown schematically in Figure 1.

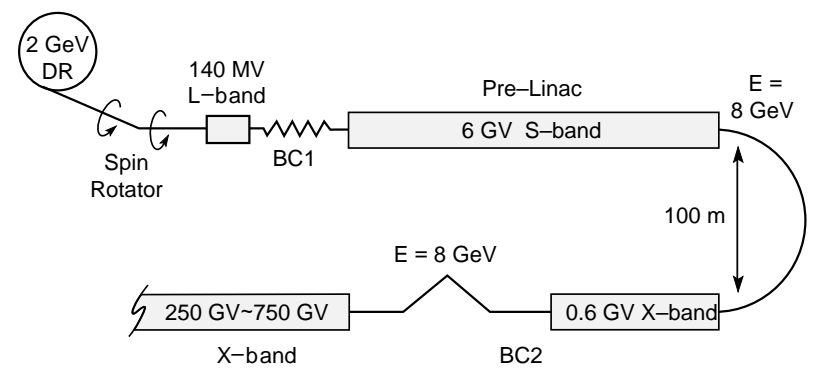

Figure 1. NLC compressor system.

The first stage of compression (BC1) is effected using a short L-band linac followed by a 10-bend wiggler. The Lband linac accelerates the beam at an RF phase of 102 degrees off crest to produce an approximately linear energy correlation of $1 \% \mathrm{rms}$ along the bunch. The magnetic wiggler, with an $R_{56}$ of $0.485-\mathrm{m}$, compresses the bunch length from $5-\mathrm{mm}$ to $0.5-\mathrm{mm}$. The $\mathrm{BC} 1$ system effectively rotates the longitudinal phase space of the beam 90 degrees, converting the long bunch-length into a finite energy spread. Table 1 lists the parameters of the first stage compressor system.

Table 1. Parameters of first compression stage.

\begin{tabular}{|l|c|c|r|}
\hline Parameter & symbol & Value & unit \\
\hline bunch population & $N$ & 7.5 & $10^{9}$ \\
bunches/train & $N_{b}$ & 95 & \\
bunch spacing & $\Delta t_{b}$ & 2.8 & $\mathrm{~ns}$ \\
ring energy & $E$ & 1.98 & $\mathrm{GeV}$ \\
DR rms energy spread & $\sigma_{\delta \mathrm{DR}}$ & 0.1 & $\%$ \\
DR rms bunch length & $\sigma_{z \mathrm{DR}}$ & 5.0 & $\mathrm{~mm}$ \\
final rms bunch length & $\sigma_{z}$ & 0.5 & $\mathrm{~mm}$ \\
final rms energy spread & $\sigma_{\delta}$ & 1.0 & $\%$ \\
length of L-band RF & $L$ & 10 & $\mathrm{M}$ \\
voltage of L-band RF & $V$ & 139 & $\mathrm{MV}$ \\
phase of L-band RF & $\varphi$ & 102 & $\mathrm{Deg}$ \\
frequency of L-band & $f$ & 1428 & $\mathrm{MHz}$ \\
$R_{56}$ of wiggler & $R_{56}$ & 0.485 & $\mathrm{M}$ \\
\hline
\end{tabular}

The compressor wiggler or chicane converts energy deviations into bunch spacing errors and vise versa. In the heavily loaded compressor linacs, it is essential to implement effective beam loading compensation schemes to remove unwanted energy deviations due to the beam loading. In addition, the $\mathrm{BC} 1$ compressor system is required to operate with beams from the damping ring that have a bunch spacing slew of 20-ps across the bunch train. The compressor RF section is required to produce a specific energy profile along the bunch train so that the bunch spacing errors will be corrected in the compressor wiggler section, preventing phase deviations in the downstream linacs.

The second stage of compression (BC2), consisting a $\mathrm{X}$-band linac followed by a chicane, compresses the bunch length from $0.5-\mathrm{mm}$ to $90-\mu \mathrm{m}$ at $8-\mathrm{GeV}$. The design issues in the $\mathrm{BC} 2$ are similar as in the $\mathrm{BC} 1$ and will not be covered here. In this paper, we will present a design for the first stage compressor. We will study the effects of the $\mathrm{BC} 1$ on the IP parameters by tracking the beams produced by the $\mathrm{BC} 1$ system through the whole linac. In these tracking studies, the $\mathrm{BC} 2$ was treated as an ideal system. This assumption was validated in separate

\footnotetext{
* Work supported by the U.S. DOE Contract No. DE-AC03-76SF00515.
} 
studies with a design of the $\mathrm{BC} 2$ that meets the requirements.

\section{THE BC1 COMPRESSOR LINAC}

The first stage compressor linac is composed of two Lband $(1.428 \mathrm{GHz})$ accelerator structures, which are identical to the $\mathrm{e}^{+}$booster accelerator structures[3], to produce a compression voltage of 140-MV along the bunch train. The linac is heavily loaded by a beam current of 1-Ampere. The beam loading energy spread must be compensated since the bunch-to-bunch energy variation will be converted into bunch spacing, or RF phase, errors by the wiggler. Unlike in the accelerator linacs, the beams in the compressor linac are approximately in phase quadrature with the RF. Under nominal operation conditions, the RF voltage does not provide compensation to the beam loading.

\subsection{Beam loading compensation schemes}

The natural choice for beam loading compensation for the compressor linac is the $\Delta \mathrm{F}$ method. However, this method requires an additional off-frequency RF system, which adds extra cost and complications.

In the present design, we adopted a $\Delta \mathrm{T}$ compensation scheme. In the $\Delta \mathrm{T}$ scheme, both phase and amplitude modulations are needed on the RF input. The phase modulation introduces an "in-phase" component of voltage to the beam that compensates the loading voltage, while the amplitude modulation is to produce the flat compression voltage along the bunch train. To obtain a phase-amplitude modulated RF waveform, we need to combine two klystrons running at different phases, but full in RF power for stability. The phase modulation of each klystron is done at low power RF control. A schematic of the $\Delta \mathrm{T}$ scheme using two phase-modulated klystrons is shown in Figure 2.

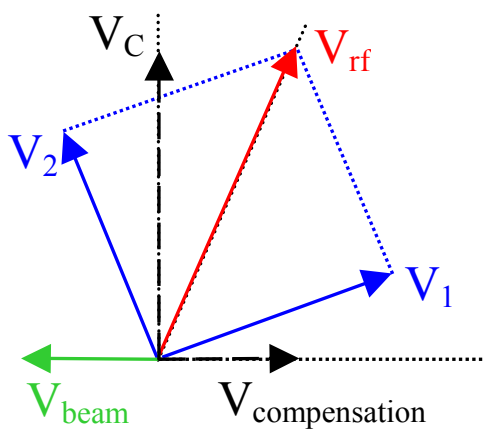

Figure 2. A schematic of $\Delta \mathrm{T}$ beam loading compensation.

The low frequency linacs in the NLC (L-band $\mathrm{e}^{+}$ booster linac and S-band pre-linacs) are powered by SLED-I[4] pulse compression systems. Although a SLED-I pulse compression system should work just fine for the compressor linacs, it is found that a pair of klystrons alone can provide enough power to drive the two accelerator sections to produce the 140-MV compression voltage. Without SLED-I pulse compression, the klystrons are required to provide 74-MW of power and $1.5-\mu$ s of pulse length. As a comparison, the system with SLED-I pulse compression would require 37-MW in klystron power and 5.5- $\mu \mathrm{s}$ in pulse length. Without pulse compression, the system is more efficient and cost effective, and is simpler to operate. The RF configuration of the L-band compressor linac is shown in Figure 3.

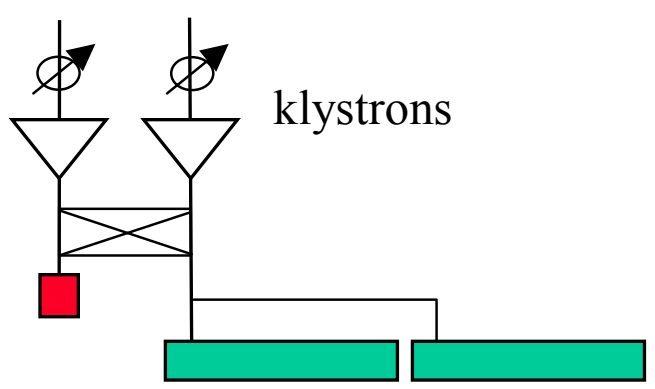

Figure 3. The L-band compressor RF configuration.

\subsection{RF profile for nominal bunch spacing}

We first optimize the RF profile for a beam with nominal bunch spacing. In the present design, the bunches are about 102 degrees off RF crest, which induces a small non-linearity term in the bunch energy profile to compensate the higher order terms (T566) in the wiggler lattice. At this phase, the RF decelerates the beam slightly in the compressor linac. After the beam is injected at one filling time $(1-\mu \mathrm{s})$ of the structure, the beam loading adds to the deceleration effect. The phase of the input RF is therefore moved toward "acceleration" to cancel the beam-loading field. In addition, the amplitude of the RF is lowered accordingly to maintain a flat compression voltage.
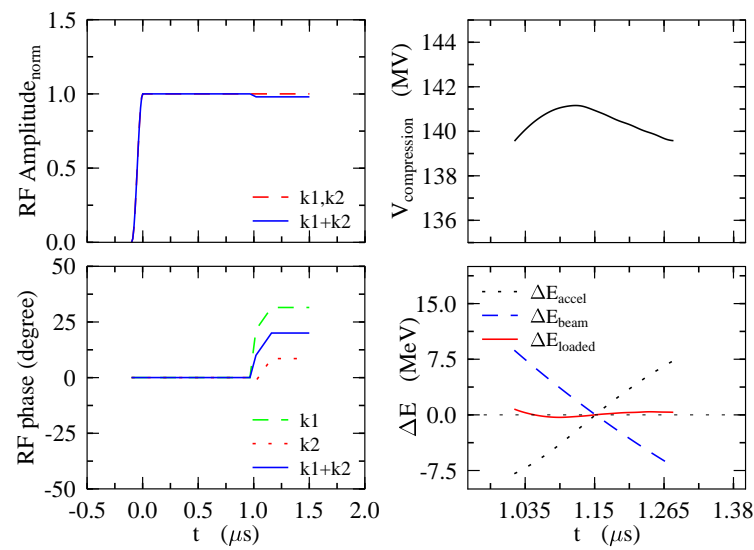

Figure 4. Beam loading compensation for the L-band compressor linac. Left: the RF pulse profile; Right: the compression voltage and energy deviation along the bunch train.

In Figure 4 are shown the RF profiles, the compression voltage and the energy deviation after compensation for a beam with nominal bunch spacing. The compression 
voltage of 140-MV is achieved with two 74-MW klystrons. The deviation of the compression voltage is less than $\pm 1 \%$. The bunch-to-bunch energy deviation is within a fraction of a MeV. The phase modulation on the klystrons is small and is obtainable using phase control at low power.

\subsection{Single and multi bunch energy profiles}

The initial 20-ps bunch-spacing slew causes the bunches to be accelerated on different RF phases. The phase $\operatorname{span}(\Delta \psi)$ due to this spacing slew (across the whole train) is one forth of the phase span of a single bunch. The energy distribution along the bunch train in the $\Delta \psi$ frame is the same as the energy profile of a single bunch. So that the bunch spacing error can be corrected the same manner as bunch length compression. An additional effect of the non-nominal bunch spacing is the phase slippage between beam loading fields of individual bunches, which results in additional non-linearity to the beam loading profile and may require additional effort to compensate. With the present beam parameters, this effect has been shown to be small in the L-band system. One can apply the same RF waveform as optimized for the nominal beams to the NLC damping ring beams.

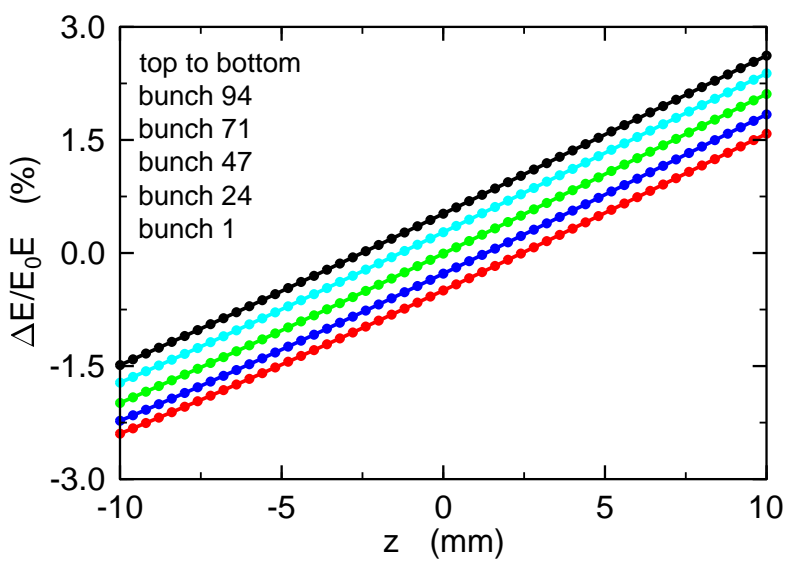

Figure 5. Energy profiles of five selected bunches along the bunch train.

Figure 5 shows the energy correlations of five bunches along the bunch train. The energy shifts among these curves are due to initial spacing errors. These shifts are converted into spacing adjustments by the wiggler, which corrects the spacing error at the exit of the compressor. As a result, there is no phase error in the S-band pre-linacs.

\section{SIMULATION RESULTS}

The stability of the longitudinal parameters over the bunch train at the IP have been studied by simulating the effect of the transient beam loading in the ring, with the added loading compensation. The nominal bunch is shown in Figure 6. The 95-bunch train is 'stretched' or 'contracted' by 17-ns (5-mm) at ring extraction and five representative bunches $(1,25,48,72$, and 95) are tracked through the entire system. The final IP energy, energy spread, bunch length, and arrival 'time' are shown versus bunch number in Figure 7.
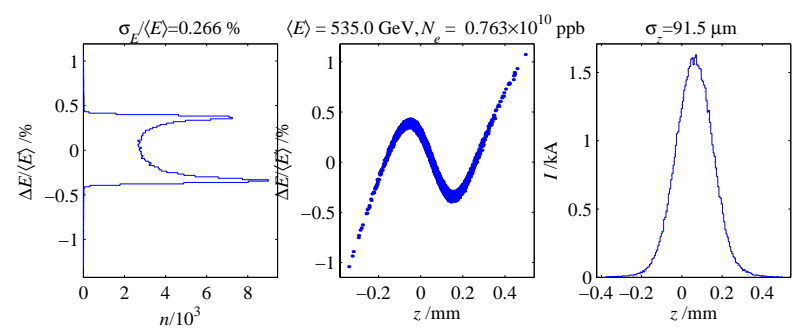

Figure 6. Energy spread (left), phase space (center), and temporal distribution (right) at IP at $535 \mathrm{GeV}$.
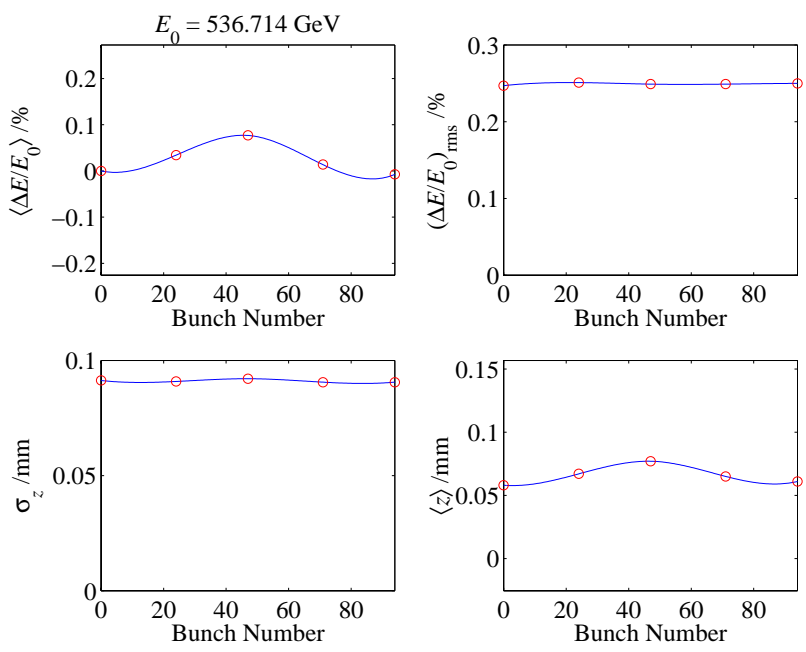

Figure 7. Final IP energy (top left), energy spread (top right), bunch length (bottom left), and relative arrival 'time' (bottom right) are shown versus bunch number.

The energy is constant over the train to a level of $<0.1 \%$, while the energy spread and bunch length are almost unaffected. The arrival 'time' of the bunch varies by $<36$-fsec $(<12-\mu \mathrm{m})$ which is $\sim 10 \%$ of the bunch length and should have no significant effect.

\section{REFERENCES}

[1] T.O. Raubenheimer, et. al., "A Bunch Compressor for the Next Linear Collider", 1995 Particle Accelerator Conference, Dallas, TX, USA, May 1995.

[2] P. Emma, "Cost and Performance Optimization of the NLC Bunch Compressor Systems", LCC-0021, Aug. 1999.

[3] Z. Li, et. al., "Parameter Optimization for the Low Frequency Linacs in the NLC," proceedings of PAC99, New York, 1999.

[4] Z. D. Farkas and et al, "SLED: A Method of Doubling SLAC's Energy," SLAC-PUB-1453, March 1974. 